\title{
Effects of exercise training alone vs a combined exercise and nutritional lifestyle intervention on glucose homeostasis in prediabetic individuals: a randomised controlled trial
}

\author{
Cris A. Slentz ${ }^{1}$ - Lori A. Bateman ${ }^{1,2}$ - Leslie H. Willis ${ }^{1}$ - Esther O. Granville ${ }^{3}$. \\ Lucy W. Piner ${ }^{1}$ - Gregory P. Samsa ${ }^{4}$. Tracy L. Setji ${ }^{5}$ Michael J. Muehlbauer ${ }^{1}$. \\ Kim M. Huffman ${ }^{1}$ - Connie W. Bales ${ }^{3}$ - William E. Kraus ${ }^{1,6}$
}

Received: 21 January 2016 / Accepted: 23 June 2016 /Published online: 15 July 2016

(C) Springer-Verlag Berlin Heidelberg 2016

\begin{abstract}
Aims/hypothesis Although the Diabetes Prevention Program (DPP) established lifestyle changes (diet, exercise and weight loss) as the 'gold standard' preventive therapy for diabetes, the relative contribution of exercise alone to the overall utility of the combined diet and exercise effect of DPP is unknown; furthermore, the optimal intensity of exercise for preventing progression to diabetes remains very controversial. To establish clinical efficacy, we undertook a study (2009 to 2013) to determine: how much of the effect on measures of glucose homeostasis of a 6 month programme modelled after the first 6 months of the DPP is due to exercise alone; whether moderate- or vigorous-intensity exercise is better for improving glucose homeostasis; and to what extent amount of exercise is a contributor to improving glucose control. The primary outcome was improvement in fasting plasma glucose, with
\end{abstract}

Cris A. Slentz

cris.slentz@duke.edu

1 Duke Molecular Physiology Institute, Department of Medicine, Duke University School of Medicine, 300 North Duke Street, Durham, NC 27701, USA

2 Present address: University of North Carolina at Chapel Hill, Center for Health Promotion and Disease Prevention, Chapel Hill, NC, USA

3 Division of Geriatrics, Duke University School of Medicine, Durham, NC, USA

4 Department of Biostatistics and Bioinformatics, Duke University School of Medicine, Durham, NC, USA

5 Division of Endocrinology, Duke University School of Medicine, Durham, NC, USA

6 Division of Cardiology, Duke University School of Medicine, Durham, NC, USA improvement in plasma glucose AUC response to an OGTT as the major secondary outcome.

Methods The trial was a parallel clinical trial. Sedentary, nonsmokers who were $45-75$ year old adults $(n=237)$ with elevated fasting glucose $(5.28-6.94 \mathrm{mmol} / \mathrm{l})$ but without cardiovascular disease, uncontrolled hypertension, or diabetes, from the Durham area, were studied at Duke University. They were randomised into one of four 6 month interventions: (1) low amount (42 kJ kg body weight ${ }^{-1}$ week $\left.^{-1}[\mathrm{KKW}]\right) /$ moderate intensity: equivalent of expending $42 \mathrm{KKW}$ (e.g. walking $\sim 16 \mathrm{~km}$ [8.6 miles] per week) with moderate-intensity $(50 \%$ $\dot{V} \mathrm{O}_{2 \text { reserve }}$ ) exercise; (2) high amount (67 KKW)/moderate intensity: equivalent of expending $67 \mathrm{KKW}(\sim 22.3 \mathrm{~km}[13.8$ miles] per week) with moderate-intensity exercise; (3) high amount (67 KKW)/vigorous intensity: equivalent to group 2, but with vigorous-intensity exercise $\left(75 \% \dot{V} \mathrm{O}_{2 \text { reserve }}\right)$; and (4) diet $+42 \mathrm{KKW}$ moderate intensity: same as group 1 but with diet and weight loss (7\%) to mimic the first 6 months of the DPP. Computer-generated randomisation lists were provided by our statistician (G. P. Samsa). The randomisation list was maintained by L. H. Willis and C. A. Slentz with no knowledge of or input into the scheduling, whereas all scheduling was done by L. A. Bateman, with no knowledge of the randomisation list. Subjects were automatically assigned to the next group listed on the randomisation sheet (with no ability to manipulate the list order) on the day that they came in for the OGTT, by L. H. Willis. All plasma analysis was done blinded by the individuals doing the measurements (i.e. lipids, glucose, insulin). Subjects and research staff (other than individuals analysing the blood) were not blinded to the group assignments.

Results Number randomised, completers and number analysed with complete OGTT data for each group were: low-amount/moderate-intensity $(61,43,35)$; high-amount/ 
moderate-intensity $(61,44,40)$; high-amount/vigorous-intensity $(61,43,38)$; diet/exercise $(54,45,37)$, respectively. Only the diet and exercise group experienced a decrease in fasting glucose $(p<0.001)$. The means and $95 \%$ CIs for changes in fasting glucose (mmol/l) for each group were: high-amount/ moderate-intensity $-0.07(-0.20,0.06)$; high-amount/vigorous $0.06(-0.07,0.19)$; low-amount/moderate $0.05(-0.05$, $0.15)$; and diet/exercise $-0.32(-0.46,-0.18)$. The effects sizes for each group (in the same order) were: $0.17,0.15$, 0.18 and 0.71 , respecively. For glucose tolerance (glucose AUC of OGTT), similar improvements were observed for the diet and exercise (8.2\% improvement, effect size 0.73 ) and the $67 \mathrm{KKW}$ moderate-intensity exercise (6.4\% improvement, effect size 0.60 ) groups; moderate-intensity exercise was significantly more effective than the same amount of vigorousintensity exercise $(p<0.0207)$. The equivalent amount of vigorous-intensity exercise alone did not significantly improve glucose tolerance (1.2\% improvement, effect size 0.21$)$. Changes in insulin AUC, fasting plasma glucose and insulin did not differ among the exercise groups and were numerically inferior to the diet and exercise group.

Conclusions/interpretation In the present clinical efficacy trial we found that a high amount of moderate-intensity exercise alone was very effective at improving oral glucose tolerance despite a relatively modest $2 \mathrm{~kg}$ change in body fat mass. These data, combined with numerous published observations of the strong independent relation between postprandial glucose concentrations and prediction of future diabetes, suggest that walking $\sim 18.2 \mathrm{~km}$ (22.3 km prescribed with $81.6 \%$ adherence in the $67 \mathrm{KKW}$ moderate-intensity group) per week may be nearly as effective as a more intensive multicomponent approach involving diet, exercise and weight loss for preventing the progression to diabetes in prediabetic individuals. These findings have important implications for the choice of clinical intervention to prevent progression to type 2 diabetes for those at high risk. Trial registration: ClinicalTrials.gov NCT00962962

Funding: The study was funded by National Institutes for Health National Institute of Diabetes and Digestive and Kidney Diseases (NIH-NDDK) (R01DK081559)

Keywords Diabetes Prevention Program - Exercise intensity · Glucose tolerance $\cdot$ Impaired fasting glucose $\cdot$ Insulin sensitivity $\cdot$ Weight-loss diet
Abbreviations
DPP
Diabetes Prevention Program
KKW $\mathrm{kJ} \mathrm{kg}$ body weight ${ }^{-1}$ week $^{-1}$
STRRIDE- Studies of Targeted Risk Reduction
[PD]

\section{Introduction}

Type 2 diabetes is one of the leading health threats in the developed world. The landmark Diabetes Prevention Program (DPP) established lifestyle changes (diet, exercise and weight loss) to be the 'gold standard' therapy for prevention of diabetes [1]. However, efficient and effective implementation of lifestyle interventions to prevent diabetes requires the following issues to be established: the relative contribution of exercise alone to the overall diet and exercise effect of DPP; whether moderate- or vigorous-intensity exercise is better for improving glucose homeostasis; and whether the amount of exercise is an important variable for improving glucose control. Resolution of these issues is important for implementation of the optimal lifestyle programme for prevention of diabetes. If providers in busy clinical practices find it necessary and effective to counsel only one component of a lifestyle intervention at a time and/or patients are more likely to undertake a single lifestyle change at a time (e.g. an exercise-only programme), it is critical to understand how much of the beneficial effects of the diet and exercise programme they are likely to expect.

The most efficacious exercise intensity for control of glucose metabolism remains controversial. Many studies suggest that moderate-intensity exercise may be more beneficial than vigorous-intensity exercise for some outcomes [2-13]. In STRRIDE (Studies of Targeted Risk Reduction Interventions through Defined Exercise), we observed that moderate-intensity training was more effective at improving insulin sensitivity [2,3], the metabolic syndrome [6], triacylglycerols [4, 13] and beta cell function [5] than an energy equivalent amount of vigorous-intensity exercise training. In contrast, one recent study [14] implies that vigorous-intensity exercise is superior to moderate-intensity exercise for glucose control. Few studies have tested head to head the effects of exercise intensity for glucose homeostasis while simultaneously controlling for total exercise energy expenditure or the effect of exercise amount while controlling for intensity.

To better understand the exercise benefit relative to a combined exercise/diet programme, and to explore the independent influence of exercise intensity and amount for improvement in glucose homeostasis in those at risk for diabetes, we undertook STRRIDE-PD (STRRIDE in individuals with Prediabetes; i.e., in individuals at risk for progression to diabetes with fasting plasma glucose of 5.3 to $6.9 \mathrm{mmol} / \mathrm{l}$ on two consecutive occasions, separated by at least 1 week), a clinical efficacy trial. The study goals meant that the study design incorporated qualitative comparisons with a diet and exercise programme modelled after the first 6 months of the DPP (rather than with a non-exercising control group). To investigate the role of intensity and amount, direct statistical comparisons were made among the exercise-only groups. Based on our previous observations - and perhaps contrary to 'common 
wisdom'-we hypothesised that: a high percentage of the diet and exercise effect on measures of glucose control would be achieved with a high amount of moderate-intensity exercise; moderate-intensity exercise would be superior to vigorousintensity exercise training, controlling for amount; and a high amount would be superior to a low amount of moderateintensity exercise.

We are not aware of any other studies that have studied the effects of different intensities of exercise training controlling for total energy expenditure on oral glucose tolerance in individuals with prediabetes.

\section{Methods}

\section{Study design}

The protocol was approved by the Duke Institutional Review Board and informed written consent obtained. Participants were recruited continuously between 2009 and 2012 from Durham (NC, USA) and the surrounding area. We randomised 237 sedentary $45-75$ year old adults with BMI $25-35 \mathrm{~kg} / \mathrm{m}^{2}$. A statistician (G. P. Samsa) provided four computer-generated randomisation lists by sex/race (white women, white men, non-white women, non-white men) in blocks of 12 . The randomisation lists were maintained by L. H. Willis and C. A. Slentz with no knowledge of, and no input into, scheduling, whereas all scheduling was done by L. A. Bateman with no knowledge of the randomisation list. Participants were automatically assigned to the next group on the randomisation list (with no ability to manipulate the list order) on the day that they came in for their glucose tolerance test by L. H. Willis. Recruited individuals had two consecutive fasting glucoses between 5.28 and $6.94 \mathrm{mmol} / 1$ taken 1 week apart. Exclusion criteria included smoking, diabetes, uncontrolled hypertension, musculoskeletal disorders and cardiovascular disease. The study design was listed on clinicaltrials.gov (registration number NCT00962962) prior to initiation of the study.

\section{Interventions}

Participants were randomised into one of four 6 month intervention groups consisting of exercise predominantly on treadmills, but also elliptical trainers, rowing and cycle ergometers: (1) low amount/moderate-intensity exercise ( $42 \mathrm{~kJ} \mathrm{~kg}$ body weight $^{-1}$ week $^{-1}[\mathrm{KKW}]$ ), exercise energy expenditure of 42 $\mathrm{KKW}$ at $50 \% \dot{V} \mathrm{O}_{2 \text { reserve }}$; (2) high amount/moderate-intensity exercise $(67 \mathrm{KKW}), 67 \mathrm{KKW}$ at $50 \% \dot{V} \mathrm{O}_{2 \text { reserve }}$; (3) high amount/vigorous-intensity exercise $(67 \mathrm{KKW}), 67 \mathrm{KKW}$ at $75 \% \dot{V} \mathrm{O}_{2 \text { reserve }}$; and (4) clinical lifestyle intervention (diet + exercise): $42 \mathrm{KKW}$ at $50 \% \dot{V} \mathrm{O}_{2 \text { reserve }}$ plus a diet designed to reduce body weight by $7 \%$ over 7 months.
$\dot{V} \mathrm{O}_{2 \text { peak }}$ was determined by a graded maximal treadmill test which started at $3 \mathrm{mph} / 0 \%$ grade and then increased speed and/or grade such that the metabolic demand increased at approximately $3.5 \mathrm{ml} \mathrm{kg}^{-1} \mathrm{~min}^{-1}$ per stage. $\dot{V} \mathrm{O}_{2 \text { reserve }}(50 \%$ or $75 \%$ ) was chosen for the exercise prescription and defined as 0.50 or $0.75 \times\left(\dot{V} \mathrm{O}_{2 \text { peak }}-\right.$ resting $\left.\dot{V} \mathrm{O}_{2}\right)+$ resting $\dot{V} \mathrm{O}_{2}$. Energy $(\mathrm{kJ})$ expended during exercise per week was determined based on the per cent of $\dot{V} \mathrm{O}_{2 \text { reserve }}$ only, without including the resting $\dot{V} \mathrm{O}_{2}$. This is the most accurate way to compare groups exercising at differing intensities [15]. The heart rate at any given intensity was determined by a progressive exercise test with gas exchange. Exercise frequency was up to the participant; a frequency was recommended with the goal of a 60 min maximum for a single session. Participants were asked to exercise under supervision on at least 2 days per week; at these sessions, we downloaded their heart rate monitors and discussed training progress. Participants were allowed to exercise at our fitness centre without supervision on the other days.

A 10 week ramp period gradually increased exercise amount to the prescribed level. Exercise intensity was maintained by using a target heart rate range confirmed by a submaximal oxygen consumption testing at the midpoint of the exercise programme. Thus, as fitness increased, participants worked harder to achieve a similar heart rate, which required reducing weekly exercise time to maintain constant weekly energy expenditure. All exercise was verified by Garmin (Olathe, KS, USA) downloadable heart rate monitors. Maximal exercise duration was capped at $6 \mathrm{~h} /$ week to limit participant burden-women have lower $\dot{V} \mathrm{O}_{2 \text { peak }}$ so had to exercise longer to expend the same total energy per week, and we believed this would have placed a disproportionate time burden on women and reduced compliance. Similarly, participants with low exercise capacity had to exercise for a longer time to achieve the desired weekly energy expenditure.

Participants randomised to the diet and exercise group received an intervention modelled after the DPP [1], designed to achieve a $7 \%$ weight reduction via energy-intake restriction, low-fat diet and exercise. Following four initial counselling sessions, participants attended 12 bi-weekly intensive group sessions adapted from the DPP manual.

Body composition Body composition was determined using air displacement plethysmography (BOD POD Life Measurement, Concord, CA, USA).

OGTT and lipids Before training and between 16 and $24 \mathrm{~h}$ after the last exercise training bout, after a $10 \mathrm{~h}$ fast, participants drank a $75 \mathrm{~g}$ glucose drink with blood samples taken at 0, 30, 60, 90 and $120 \mathrm{~min}$. Participants were asked to eat their normal diet the evening before each test. Glucose, total cholesterol, HDL-cholesterol and triacylglycerol were measured 
with a Beckman-Coulter DxC600 clinical analyser (Brea, CA, USA). Insulin was measured by electrochemiluminescent plate assay, Meso Scale Discovery (Gaithersburg, MD, USA). AUCs of the glucose and insulin curves were calculated by the trapezoid method. Matsuda index was calculated as described in Matsuda and Defronzo [16].

Outcomes The primary outcome for this trial was fasting glucose. Other strong secondary outcomes included other measures of glucose control: specifically, changes in glucose tolerance (AUCs for glucose, and $1 \mathrm{~h}$ and $2 \mathrm{~h}$ glucose values), fasting insulin and insulin AUC. Additional outcomes included measures of cardiometabolic health (lipids, waist circumference, cardiorespiratory fitness, body weight and body composition).

Sample size Based on our experience from STRRIDE [2, 3], we estimated we would need 35-45 finishers per group to have adequate power to detect significant changes within groups for cardiometabolic variables and that this sample size would provide enough power to detect differences between groups. While neither we nor others have specific data on the improvement in glucose tolerance expected for different exercise intensities, the study by Houmard et al [3] had adequate power to detect a difference in insulin sensitivity between the low amount/moderate intensity and the low amount/vigorous intensity groups $(p<0.05)$, which strongly supported an intensity effect (i.e. moderate intensity was superior). While we hoped to finish with 50 participants in each group, funding cuts meant we had to stop just short of that goal (within 10\%).

Statistical analyses A priori, the study was designed to make only qualitative comparisons (per cent comparisons, nonstatistical) among the three exercise-only groups when compared with the diet, exercise and intentional weight loss group; therefore, there was no inactive control group in this study. To address the question of how the exercise-only interventions compared with the lifestyle intervention, percentages of the effect observed with each of exercise-only intervention relative to that obtained with the diet-exercise group is reported. This is justified based on the findings of DPP, in which the lifestyle intervention was superior to metformin and control [1]; therefore, it was not clinically useful to compare the effects of our interventions with a control.

The primary quantitative comparisons were assessing the independent effects of exercise intensity and amount on outcomes. The study was powered for two statistical comparisons: (1) an exercise-intensity effect was tested by comparing the two $67 \mathrm{KKW}$ groups (high amount/moderate vs highamount/vigorous); and (2) an exercise amount effect was tested by comparing the two moderate-intensity groups. Group comparisons were performed with ANCOVA and included baseline values as covariates (Statview, Cary, NC, USA). Post hoc analyses were confined to the two comparisons among the exercise groups (of intensity and amount): the critical $\alpha$ was $p<0.025$ to account for two major comparisons. To determine whether within-group changes (i.e. not between groups) were significant, we used two-tailed paired (pre-intervention vs post-intervention) $t$ tests, where critical $\alpha$ was $p<0.05$. The analyses presented were performed 'per protocol'.

\section{Results}

Figure 1 shows the flow diagram of screening, randomisation and dropout numbers. Demographic baseline characteristics and exercise prescriptions are presented in Table 1. Baseline values and change scores for body composition and metabolic variables are shown in Table 2. The two high-amount groups were significantly older than the low-amount and diet/exercise groups. There were no other baseline differences between groups for variables in Tables 1 or 2. Participants who dropped out were heavier (92.2 vs $87.2 \mathrm{~kg}$ ), had greater fat mass (38.0 vs $35.5 \mathrm{~kg}$ ) and larger waist circumferences (102.8 vs $99.2 \mathrm{~cm}$ ) compared with the completers at baseline (all $p<0.05$, data not shown). No other differences between completers and dropouts were observed for variables presented in Tables 1 and 2. There were no differences for any metabolic outcome variable at baseline between intervention groups or between completers and dropouts.

Exercise prescriptions are in Table 1. The time required to expend $67 \mathrm{KKW}$ is based on fitness levels (oxygen consumption rate; $\mathrm{ml} \mathrm{kg}^{-1} \mathrm{~min}^{-1} ; 11 \mathrm{O}_{2}$ consumption is roughly equivalent to $21 \mathrm{~kJ}$ energy use) and exercise training intensity (moderate or vigorous). Individuals with lower maximal fitness levels required more time to complete the energyexpenditure-based exercise prescriptions at either $50 \%$ or $75 \%$ of this value. While energetically equivalent, moderateintensity exercise appears to improve some key metabolic variables more than vigorous-intensity, vigorous-intensity exercise improved fitness levels to a greater degree.

Table 2 presents baseline and change scores for key variables. As expected, all groups lost weight (though the weight lost by the low amount/moderate intensity group was not significant). Also, when expressed in $\mathrm{ml} \mathrm{kg}^{-1} \mathrm{~min}^{-1}$, the diet/exercise group and both high-amount exercise groups experienced significant improvements in cardiorespiratory fitness. Importantly, this increase in fitness was due entirely to weight loss for the diet/exercise group, because when expressed as $1 / \mathrm{min}$ there was no increase for this group. Changes in body composition were greatest for the diet/exercise group; and for the exercise-only groups, corresponded to total exercise energy expenditure. 




Fig. 1 Screening flowchart

Magnitude of exercise contribution Only the diet/exercise group experienced significant reductions in fasting glucose (Table 2). Diet/exercise also had the most robust effects on fasting insulin, HOMA, Matsuda index and insulin AUC. As indicated by changes in insulin AUC and Matsuda index score, all interventions improved insulin sensitivity. For improving glucose tolerance, the low amount/moderate intensity group was half as effective as diet/exercise (53\%) and twice as effective as the high amount/vigorous intensity group.

Intensity effects For both the diet/exercise and the high amount/moderate intensity group, changes in glucose AUC were similar and quite robust (Fig. 2a). The high amount/ moderate-intensity exercise group achieved $79 \%$ of the improvement in glucose tolerance of the diet/exercise group ( $t$ test for within-group change for high/moderate group, $p=0.0005$; Table 2). The glucose incremental AUC data show that the main difference between lifestyle and high amount/ moderate was due to the decrease in fasting glucose by the lifestyle group. The AUC incremental improvement was actually higher for the high/moderate group.

In contrast, the high amount/vigorous intensity group experienced a very small, non-significant, change in glucose
AUC ( $t$ test, $p=0.20)$. Importantly, the high amount/moderate intensity group was statistically superior to the high amount/ vigorous group ( $p=0.0207$; effect size $=0.445$; Fig. $2 \mathrm{a}$ ). The low amount/moderate intensity group was intermediate between the high-amount exercise groups; however, the improvement was not quite significant $(p<0.10)$.

Amount effects The high amount/moderate intensity group experienced larger quantitative improvements than the low amount/moderate-intensity exercise group for most, but not all, of the glucose homeostasis variables (Fig. 2a-d, Table 2), although there were no statistically significant differences between the two moderate-intensity groups. The effects of the different interventions on the glucose and insulin curves are shown in Fig. 3a-d (glucose) and Fig. 4a-d (insulin). Glucose values at 30,60 and 90 but not 120 min were significantly improved after exercise training in the high amount/moderate intensity group, whereas the reverse occurred in the high amount/vigorous intensity group (Fig. 3a). When a separate ANCOVA was performed between the high amount/moderate vs high amount/vigorous groups on each time point $(30,60,90$ and $120 \mathrm{~min}$ ) the $p$ values for statistical comparisons at these four time points were: $0.035 ; 0.086,0.081$ and 0.597 , 
Table 1 Baseline demographics and exercise prescriptions

\begin{tabular}{|c|c|c|c|c|c|}
\hline Variable & $\begin{array}{l}\text { Total group } \\
(n=150)\end{array}$ & $\begin{array}{l}\text { High amount/moderate } \\
\text { intensity }(67 \mathrm{KKW} \text { at } \\
50 \%)(n=40)\end{array}$ & $\begin{array}{l}\text { High amount/vigorous } \\
\text { intensity }(67 \mathrm{KKW} \text { at } \\
75 \%)(n=38)\end{array}$ & $\begin{array}{l}\text { Low amount/moderate } \\
\text { intensity }(42 \mathrm{KKW} \text { at } \\
50 \%)(n=35)\end{array}$ & $\begin{array}{l}\text { Diet }+ \text { low amount/ } \\
\text { moderate intensity } \\
(42 \mathrm{KKW} \text { at } \\
50 \%)(n=37)\end{array}$ \\
\hline Age & $59.1(7.7)$ & $61.4(7.1)$ & $60.4(7.0)$ & $56.9(7.8)$ & $57.6(8.1)$ \\
\hline BMI $\left(\mathrm{kg} / \mathrm{m}^{2}\right)$ & $30.3(2.6)$ & $30.0(2.4)$ & $30.0(2.6)$ & $30.6(2.3)$ & $30.9(2.8)$ \\
\hline$\dot{V} \mathrm{O}_{2 \text { peak }}\left(\mathrm{ml} \mathrm{kg}^{-1} \min ^{-1}\right)$ & $24.6(5.2)$ & $24.5(5.1)$ & $23.4(5.3)$ & $24.9(5.7)$ & $25.0(4.6)$ \\
\hline \multicolumn{6}{|l|}{ Race } \\
\hline White & $121(80.7 \%)$ & 35 & 30 & 28 & 28 \\
\hline African-American & $22(14.7 \%)$ & 5 & 5 & 7 & 5 \\
\hline Other & $7(4.7 \%)$ & 0 & 3 & 0 & 4 \\
\hline \multicolumn{6}{|l|}{ Sex } \\
\hline Women & $88(58.7 \%)$ & 23 & 23 & 22 & 20 \\
\hline Men & $62(41.3 \%)$ & 17 & 15 & 13 & 17 \\
\hline \multicolumn{6}{|l|}{ Exercise prescription } \\
\hline Intensity $\left(\% \dot{V} \mathrm{O}_{2 \text { peak }}\right)$ & & 50 & 75 & 50 & 50 \\
\hline Exercise amount (KKW) & & 67 & 67 & 42 & 42 \\
\hline Exercise time (min per week) & & $287(54)$ & $195(38)$ & $181(35)$ & $175(35)$ \\
\hline Women & & $317(43)$ & $217(25)$ & $199(26)$ & $188(32)$ \\
\hline Men & & $246(40)$ & $161(28)$ & $151(39)$ & $160(33)$ \\
\hline \multicolumn{6}{|l|}{ Actual exercise results } \\
\hline Adherence ( $\%$ of prescribed min) & & $81.6(16)$ & $85.2(17)$ & $85.1(21)$ & $85.8(18)$ \\
\hline Frequency (sessions/week) & & $3.9(0.9)$ & $3.2(0.7)$ & $3.1(0.7)$ & $2.9(0.7)$ \\
\hline Amount completed (KKW) & & $54.8(10.5)$ & $56.8(11.7)$ & $35.5(8.8)$ & $35.9(7.5)$ \\
\hline $\begin{array}{l}\text { Approx. amount done } \\
\text { (miles per week) }\end{array}$ & & $11.3(2.1)$ & $11.7(2.4)$ & $7.3(1.8)$ & $7.4(1.5)$ \\
\hline Average heart rate (bpm) & & $113.4(10.4)$ & $127.4(13.1)$ & 116.9 (12.6) & $116.7(12.7)$ \\
\hline Average speed $(\mathrm{km} / \mathrm{h})$ & & $5.2(0.6)$ & $5.6(0.8)$ & $5.090 .8)$ & $5.0(1.0)$ \\
\hline Average grade $(\%)$ & & $3.7(1.8)$ & $5.3(2.8)$ & $3.6(1.5)$ & $3.6(2.2)$ \\
\hline Actual time $(\mathrm{min} / \text { week })^{\mathrm{d}}$ & & $230(54)$ & $165(43)$ & $157(41)$ & $150(39)$ \\
\hline Women & & $247(52)$ & $176(47)$ & $170(33)$ & $158(40)$ \\
\hline Men & & $207(50)$ & $148(32)$ & $134(45)$ & $140(35)$ \\
\hline
\end{tabular}

Values are means (SD) unless otherwise indicated

Prescribed exercise amounts (42 and $67 \mathrm{KKW}$ ) are approximately equivalent to 8.6 and 13.8 miles of walking/jogging per week

Amount completed $=$ exercise amount $\times$ adherence

Actual time $(\mathrm{min} /$ week $)=$ exercise time $\times$ adherence

As exercise prescription is made equivalent per unit of body mass, the only factors that determines the amount of time required to expend the appropriate number of kJ is fitness $\left(\dot{V} \mathrm{O}_{2 \text { peak }}\right)$ and number of KKW (i.e. in this study, either 42 or $\left.67 \mathrm{KKW}\right)$; this explains why women, who have on average lower fitness levels when expressed per kg body weight, have a higher number of prescribed min for any given exercise prescription, when compared with men

respectively. The high amount of moderate-intensity exercise resulted in significant improvements in glucose at $30 \mathrm{~min}$. Though the high amount/vigorous group experienced a significant improvement in glucose at $120 \mathrm{~min}$, this improvement was not different from the improvement in the high amount/ moderate intensity group. As was expected, the largest and most consistently significant improvement was observed in the clinical lifestyle group. As illustrated in Fig. 4a-d, all four interventions resulted in robust reductions in overall insulin levels and in reductions in insulin at all time points.

\section{Discussion}

There were two major goals for our study: (1) to determine, in individuals at risk for diabetes, what percentage of the 'gold standard' effect of diet and exercise can be achieved with aerobic exercise alone on glucose homeostasis measures (fasting glucose, oral glucose tolerance and measures of insulin sensitivity); and (2) to determine the independent effects of exercise intensity and amount on these same measures in individuals at risk of developing diabetes. We are not aware of any other studies that have investigated the effects of different intensities of exercise training on oral glucose tolerance in individuals with prediabetes. For the primary outcome, fasting glucose, there was significant improvement only in the diet and exercise group. The exercise-only groups did not experience any change in fasting glucose. With respect to glucose tolerance, there were two major findings. First, the effect of a high amount/moderate-intensity exercise on glucose tolerance was essentially the same as that for the diet/exercise group; this exercise-only group achieved $79 \%$ of the improvement in 
Table 2 Baseline values and change scores for body composition, fitness, lipids and measures of glucose control

\begin{tabular}{|c|c|c|c|c|c|c|c|c|c|c|c|c|}
\hline \multirow[t]{2}{*}{ Variable } & \multicolumn{3}{|c|}{$\begin{array}{l}\text { High amount/moderate } \\
\text { intensity } 67 \mathrm{KKW} \text { at } 50 \% \\
\dot{V} \mathrm{O}_{2 \text { peak }}(n=40)\end{array}$} & \multicolumn{3}{|c|}{$\begin{array}{l}\text { High amount/vigorous } \\
\text { intensity } 67 \mathrm{KKW} \text { at } 75 \% \\
\dot{V} \mathrm{O}_{2 \text { peak }}(n=38)\end{array}$} & \multicolumn{3}{|c|}{$\begin{array}{l}\text { Low amount/moderate } \\
\text { intensity } 42 \mathrm{KKW} \text { at } 50 \% \\
\dot{V} \mathrm{O}_{2 \text { peak }}(n=35)\end{array}$} & \multicolumn{3}{|c|}{$\begin{array}{l}\text { Clinical lifestyle } \\
\text { intervention Low amount/ } \\
\text { moderate intensity }+ \\
\operatorname{diet}(n=37)\end{array}$} \\
\hline & Baseline & Change & Sig. & Baseline & Change & Sig. & Baseline & Change & Sig. & Baseline & Change & $\mathrm{Si}$ \\
\hline ody & ) & -1.9 & $* * *$ & 8 & -1.6 & & 86 & -0 & $\dagger$ & 5) & $5.1)$ & \\
\hline Fat ma & 2) & -2.2 & $* * *$ & 34.4 & -2.3 & & .1) & -1.1 & $\dagger$ & $8)$ & & \\
\hline Lean $\mathrm{m}$ & $.6(11)$ & 0.26( & & 49.3 & 0.66 & $*$ & 51.7 & $0.19(1.9)$ & & $53.8(12)$ & $-0.46(2.4)$ & \\
\hline Waist circumference $(\mathrm{cm})$ & $.1(9)$ & $-1.39(2.6)$ & $* *$ & $99.2(8)$ & $-1.63(2.3)$ & $* * *$ & $98.8(8)$ & $-0.82(2.8)$ & & $99.5(10)$ & $-4.54(4.9)$ & $* * *$ \\
\hline$\dot{V} \mathrm{O}_{2 \text { peak }}\left(\mathrm{ml} \mathrm{kg}^{-1} \mathrm{~min}^{-1}\right)$ & $24.6(5)$ & $1.5(2)$ & $* * *$ & $24.3(5)$ & $2.6(3)$ & $* * *$ & $24.8(6)$ & $0.9(3)$ & $\dagger$ & $25.5(4)$ & $2.8(3)$ & $* * *$ \\
\hline$\dot{V} \mathrm{O}_{2 \text { peak }}(1 / \mathrm{min})$ & $2.19(0.7)$ & $0.08(0.3)$ & $\dagger$ & $2.11(0.7)$ & $0.18(0.4)$ & $* *$ & $2.21(0.7)$ & $0.02(0.3)$ & & $2.37(0.7)$ & $-0.01(0.2)$ & \\
\hline $\begin{array}{l}\dot{V} \mathrm{O}_{2 \text { peak }} \\
\quad\left(\mathrm{ml} \mathrm{kg} \mathrm{lbm}{ }^{-1} \mathrm{~min}^{-1}\right)\end{array}$ & $42.0(7.0)$ & $1.75(4.9)$ & $\dagger$ & $41.2(8.4)$ & $3.06(7.4)$ & $*$ & $43.6(8.2)$ & $-0.15(6.4)$ & & $43.8(8.0)$ & $0.43(5.5)$ & \\
\hline Total cholesterol $(\mathrm{mmol} / \mathrm{l})$ & $4.90(0.8)$ & $-0.002(0.5)$ & & $4.64(0.7)$ & $-0.018(0.4)$ & & $4.767(1.0)$ & $0.012(0.6)$ & & $4.96(0.8)$ & $-0.24(0.6)$ & $*$ \\
\hline HDL-cholesterol (mmol/l) & $1.13(0.4)$ & $0.02(0.1)$ & & $1.16(0.3)$ & $0.06(0.1)$ & $* *$ & $1.19(0.6)$ & $-0.89(0.3)$ & & $1.09(0.3)$ & $0.06(0.2)$ & $*$ \\
\hline LDL-cholesterol (mmol/l) & $3.01(0.7)$ & $-0.02(0.5)$ & & $2.77(0.6)$ & $0.07(0.5)$ & & $2.93(0.8)$ & $0.08(0.5)$ & & $2.95(0.6)$ & $-0.15(0.5)$ & $\dagger$ \\
\hline Triacylglycerol (mmol/l) & $1.64(1.1)$ & $-0.10(0.9)$ & & $1.48(0.8)$ & $-0.16(0.5)$ & $*$ & $1.42(0.6)$ & $-0.05(0.5)$ & & $1.39(0.6)$ & $-0.34(0.4)$ & \\
\hline Fasting glucose (mmol/l) & $5.91(0.5)$ & $-0.07(0.4)$ & & $5.79(0.5)$ & $0.06(0.4)$ & & $5.87(0.6)$ & $0.05(0.3)$ & & .6) & $-0.32(0.4)$ & \\
\hline Fasting insulin (pmol/l) & $45.3(31)$ & $-6.4(24)$ & $\dagger$ & 49 & -9.3 & * & $48.4(24)$ & -5.4 & $\dagger$ & 50 & -21 & ** \\
\hline HOMA & $1.81(1.3)$ & $-0.28(1.0)$ & $\dagger$ & $1.99(1.9)$ & $-0.36(1.0)$ & * & 1.93 & $-0.20(0.7)$ & $\dagger$ & .2) & $-0.90(0.9)$ & $* * *$ \\
\hline $\begin{array}{l}\text { Glucose AUC } \\
\quad(\mathrm{mmol} / 1 \times 120 \mathrm{~min})\end{array}$ & $1042(192)$ & $-73(123)$ & $* * *$ & $1031(211)$ & $-22(103)$ & & $1039(219)$ & $-49(143)$ & $\dagger$ & $1050(232)$ & $-96(132)$ & ** \\
\hline Glucose AUC incremental & $333(1$ & $-66(1$ & $* * *$ & & $-29(11$ & & & -55( & $*$ & & -58 & $* *$ \\
\hline $\mathrm{C}(\mathrm{pmol} / \mathrm{l} \times 2 \mathrm{~h})$ & $990(666)$ & $-264(452)$ & $* * *$ & 1135 (999) & $-246(452)$ & $* *$ & 955 & $-166(330)$ & $* *$ & $982(527)$ & $-348(350)$ & $* * *$ \\
\hline $1 \mathrm{~h}$ glucose $(\mathrm{mmol} / \mathrm{l})$ & $9.78(2.2)$ & $-0.82(1.8)$ & $* *$ & $9.72(2.3)$ & $-0.21(1.4)$ & & $9.67(2.6)$ & $-0.72(2.1)$ & $*$ & $9.89(2.7)$ & $-0.97(1.8)$ & $* *$ \\
\hline $\mathrm{amol} / \mathrm{l})$ & $7.52(2.4)$ & $-0.47(2.1)$ & & $8.31(2.3)$ & $-0.72(1.4)$ & $* *$ & $8.01(2.2)$ & $-0.39(1.6)$ & & $8.23(2.6)$ & $-1.30(1.9)$ & $* * *$ \\
\hline Matsuda index & $5.55(3.5)$ & $1.35(2.7)$ & $* *$ & $5.25(3.0)$ & $1.38(2.4)$ & $* * *$ & $5.14(3.2)$ & $1.08(1.7)$ & $* * *$ & $4.99(3.3)$ & 3.98 (3.9) & $* * *$ \\
\hline
\end{tabular}

Values are means (SD)

There were no significant baseline group differences for any of the variables above; all tests for significance shown in this table are for 'within-group' changes. No-between group analyses are shown

Significant change score (post-training - pre-training value) using a two-tailed $t$ test: $\dagger p<0.10 ; * p<0.05 ; * * p<0.01 ; * * * p<0.001$

Temporary breakdown of the Bod Pod machine meant that body composition data were not available for all participants at pre- and post-training time points; body composition data is based on $n=32,30,35,37$. Glucose and insulin AUCs included only participants with all five time points during the OGTT, $n=40,38,35,37$ for high/moderate, high/vigorous, low-moderate and lifestyle groups. LBM, lean body mass

glucose tolerance that was obtained with the combined therapy group. Second, high amount/moderate-intensity exercise robustly improved glucose homeostasis and had a statistically superior effect on AUC for glucose during an OGTT compared with the same amount of vigorous-intensity exercise.

These observations are remarkable. They confirm and build on our earlier findings regarding exercise training intensity effects. In STRRIDE [17], a large randomised controlled trial, we observed that, compared with vigorous-intensity exercise, the same amount of moderate-intensity exercise training led to greater improvements in: insulin sensitivity [2, 3], the metabolic syndrome [6], triacylglycerols [13] and beta cell function [5]. That is, while we [18] and others have observed that vigorous intensity is clearly superior for improvements in cardiorespiratory fitness, our previous findings, taken together with the current study, suggest that moderate-intensity exercise may be better than vigorous-intensity exercise for maintaining cardiometabolic health and preventing the development of diabetes in at-risk individuals.
Recently, in a large and well-designed exercise study [14], Ross and colleagues reached an opposing conclusion that vigorous-intensity exercise outperformed moderateintensity exercise in measures of glucose control; however, most of their observations were remarkably similar to those made in our study. In the study by Ross et al, 300 abdominally obese individuals unscreened for baseline glucose abnormalities were randomised to high amount/vigorous intensity, high amount/moderate intensity, low amount/ moderate intensity or control group; this was very similar to our study design. In the study by Ross et al, changes in waist circumference and the $2 \mathrm{~h}$ glucose time point during the OGTT were considered primary outcomes. The vigorous intensity/high amount group outperformed the controls at the 120 min OGTT glucose time point. The effect for the moderate intensity/high amount group was nonsignificant at this time point; however, direct comparison of intensity effects controlling for amount in Ross's Table 3 reveals that the effects of the high amount/vigorous and 
a
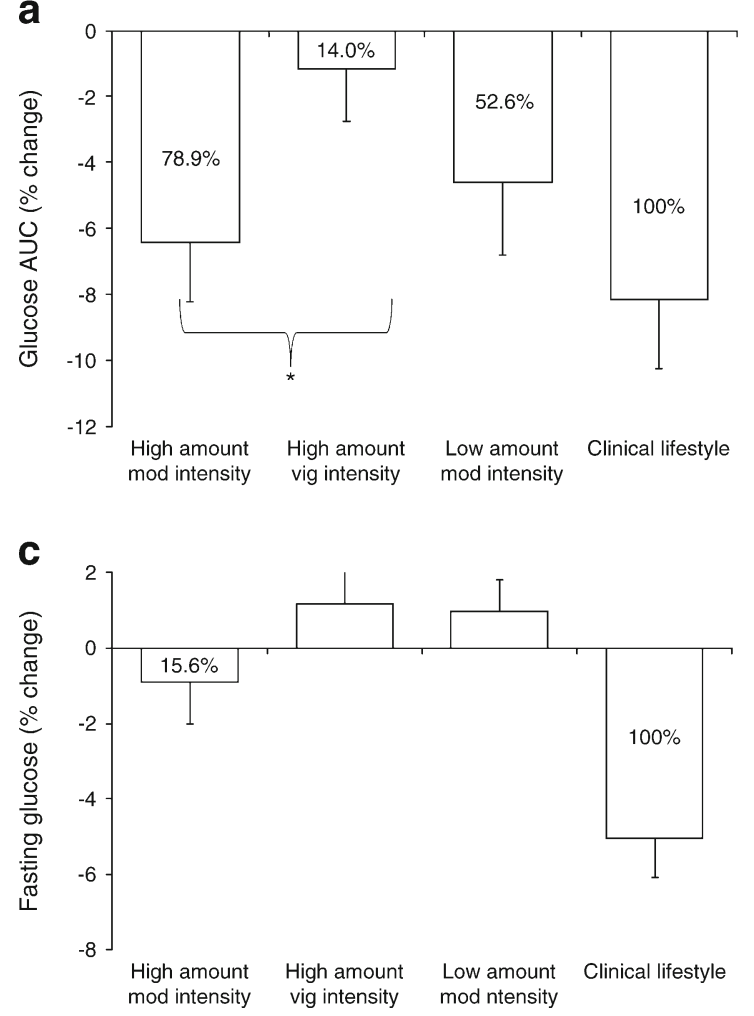

Fig. 2 Changes in glucose AUC (a), insulin AUC (b), fasting glucose (c) and fasting insulin (d) for each intervention group. The percentages inside each bar represent the percentage of the diet, exercise and intentional weight loss group (lifestyle) that was obtained for that variable, with lifestyle represented as $100 \%$. ANCOVA with baseline values as a covariate, with post hoc testing between the two groups differing in exercise intensity and between the two groups differing in amount (controlling for

high amount/moderate exercise on $2 \mathrm{~h}$ glucose were not different. Inspection of our Fig. $3 \mathrm{c}$ and Table 2 also reveal that, of the exercise-only interventions, only with vigorousintensity exercise did we observe a significant change in $2 \mathrm{~h}$ glucose. One should note that in the study by Ross et al, both high-amount groups improved several health-related measures compared with controls: insulin AUC, Matsuda index, HDL-cholesterol, fasting insulin, HOMA, and systolic and diastolic blood pressure (Appendix and Table 3 from the Ross paper). However, in Ross's study, only the high amount/moderate-intensity exercise (and not vigorous intensity) was significantly different from the control group for these measures. Thus, despite some differences in the study population and approach, our findings - if not our conclusions - are remarkably concordant with those in the Ross study. The exception is a substantial difference with our findings of a significant difference between the two high-amount groups on improvement in AUC for glucose. This may be due to the different participant populations. In the present study, participants were recruited specifically to select for abnormal glucose; in the Ross study, participants were selected for abdominal obesity. In summary the b

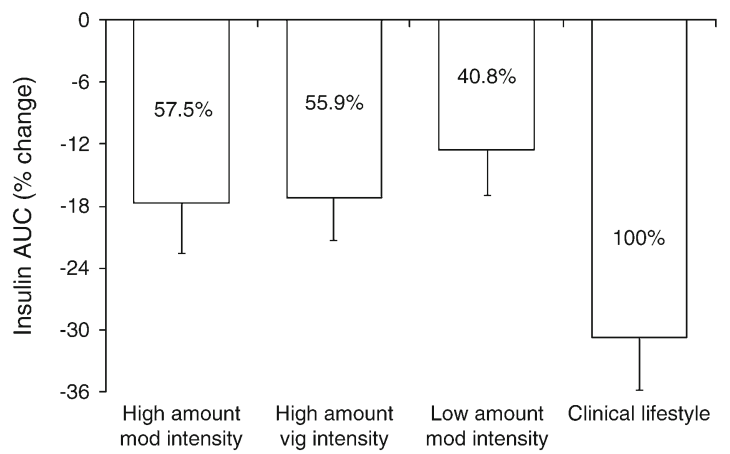

d

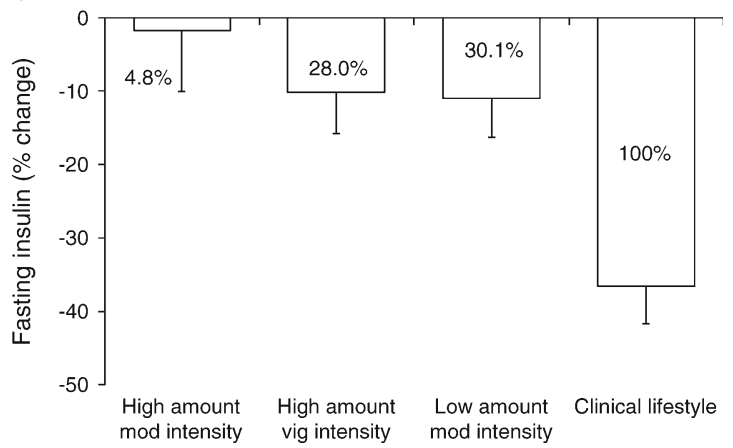

baseline values) revealed a statistically significant difference $(* p<0.05$ $[p=0.0207])$ between the high amount/moderate intensity and the high amount/vigorous intensity groups for glucose AUC. The improvement in the high amount/moderate intensity group was superior to, but not statistically different from, the low amount group. Mod, moderate; vig, vigorous

outcome of the dispute may depend on which variable is chosen as the primary outcome measure.

We conclude that the large improvement in post-load glucose observed with high amount/moderate-intensity exercise suggests this may be a very effective intervention for reducing the risk of diabetes. Several studies have observed that postload glucose is superior to fasting glucose for predicting allcause mortality, cardiovascular disease incidence and mortality, and incident diabetes [19-32]. In the San Antonio Heart Study [32] and the Botnia Study [29], $1 \mathrm{~h}$ glucose was the best single predictor of incident diabetes, significantly better than both fasting and $2 \mathrm{~h}$ glucose $[30,29]$; this is particularly relevant, as moderate-intensity exercise appears especially effective at lowering $1 \mathrm{~h}$ glucose (Fig. 3a).

The diet/exercise group experienced a robust reduction in fasting glucose, while the exercise-only groups had no effect on fasting glucose; this was an expected finding. It is intriguing to speculate about the mechanisms mediating this effect. Impaired fasting glucose is generally considered to be indicative of hepatic insulin resistance, whereas impaired glucose tolerance is considered to be reflective of peripheral insulin resistance [33]. While we did not statistically compare the diet 
Fig. 3 OGTT glucose curves. The pre- and post-training glucose means and standard error bars are plotted for each time point of the OGTT for each group: high amount/moderate intensity (a); high amount/ vigorous intensity (b); low amount/moderate intensity (c); and clinical lifestyle (d). Pretraining data are shown with solid lines, post-training data are shown with dashed lines. Significant differences for each within-group time point (pre- vs post-training value) were determined by a twotailed paired $t$ test: $* p<0.05$; ${ }^{* *} p<0.01 ; * * * p<0.001$ a
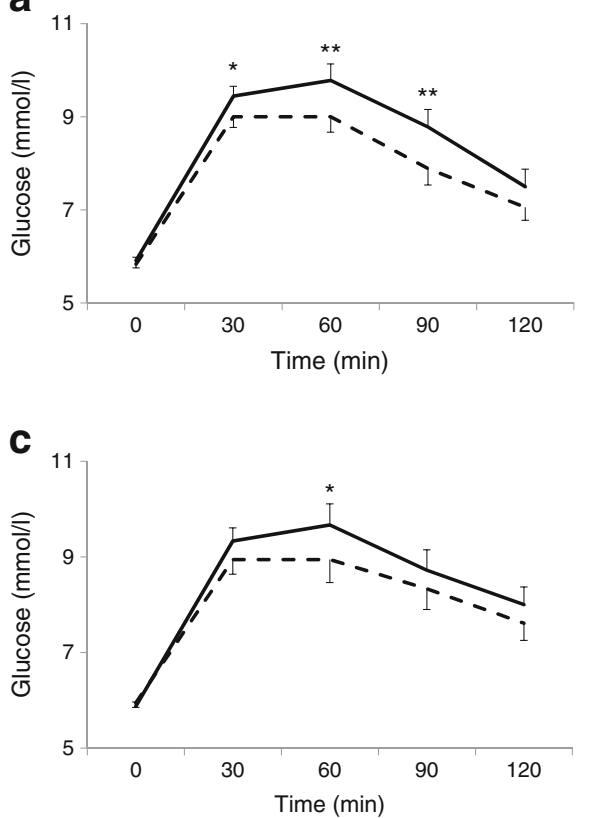

b

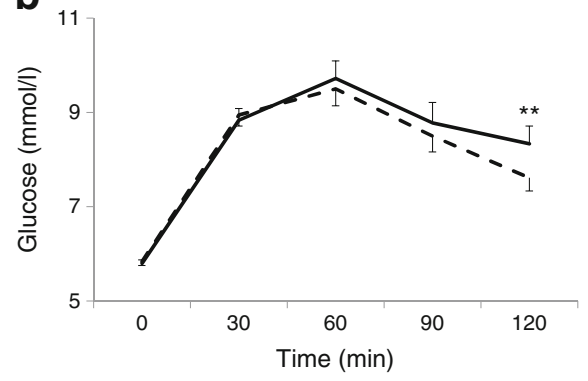

d



and exercise group with the exercise-only groups, the diet and exercise group experienced between $188 \%$ and $269 \%$ greater improvement in the Matsuda index (Table 2) than the exercise-only groups. The Matsuda index includes all glucose and insulin values obtained from the $2 \mathrm{~h}$ glucose test; thus, the robust improvements seen in these individual variables by the diet/exercise group was reflected in the Matsuda index.

We were surprised to observe that vigorous-intensity exercise training had no significant effect on glucose AUC when measured 16-24 h after the last exercise training bout. While this group experienced reductions in insulin AUC, it will be important to understand (through future mechanistic studies) why vigorous-intensity training did not improve glucose tolerance. Perhaps vigorous-intensity exercise-but not moderate-intensity exercise-results in increases in glucose counter-regulatory hormones or skeletal muscle adaptations to acute exercise that prevent the maximum benefit of training to be accrued between 16 and $24 \mathrm{~h}$ after the last bout of exercise.

It is important to note the limitations of this study. Obtaining a high amount of moderate-intensity exercise in individuals with low fitness is challenging. Participants in
Fig. 4 OGTT insulin curves. The pre-training and post-training insulin means and standard error bars are plotted for each time point of the OGTT, for each intervention group: high amount/ moderate intensity (a); high amount/vigorous intensity (b); low amount/moderate intensity (c); and clinical lifestyle (d). Pretraining data are shown with solid lines, post-training data are shown with dashed lines. Significant differences for each within-group time point (pre- vs post-training value) were determined by a twotailed paired $t$ test: ${ }^{\dagger} p<0.10$; ${ }^{*} p<0.05 ; * * p<0.01$;

$* * * p<0.001$ a

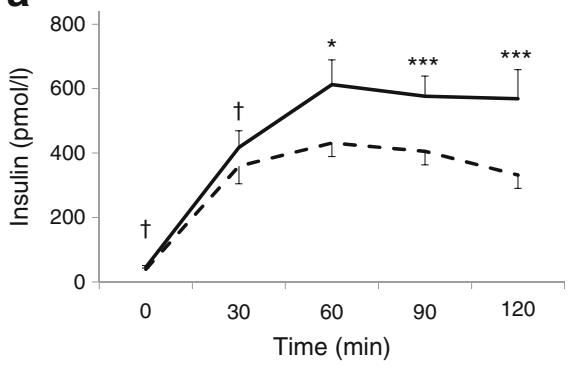

C



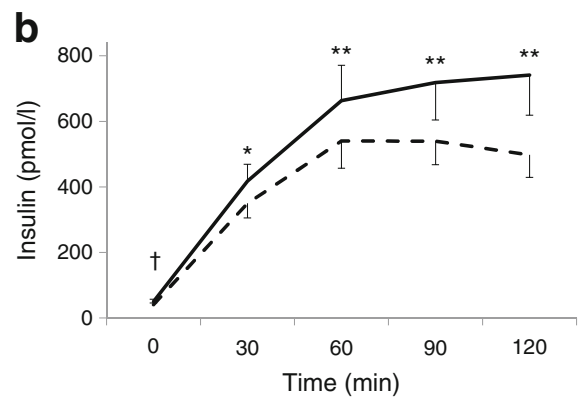

d

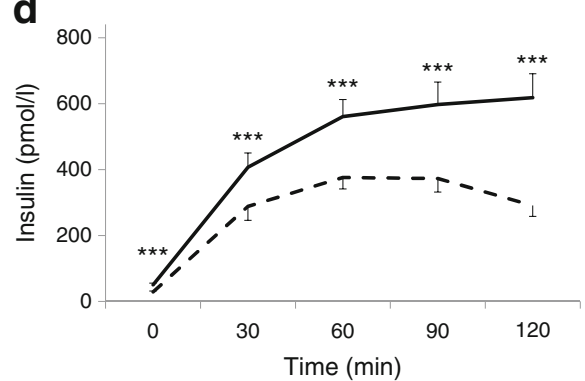


our study required, on average, $4.8 \mathrm{~h}$ of moderate-intensity exercise (more for women) to expend $67 \mathrm{KKW}$. Importantly, the diet part of the diet/exercise weight loss group was much more intensive and included many additional one-on-one and small-group interactions; this resulted in the lowest dropout rate of all of the groups. We did not control for the greater amount of personal contact time received by the diet/exercise relative to the exercise-only groups. Finally, we can only infer the effects of the different interventions on true diabetes incidence and progression. In reality, only a diabetes outcome study would be able to determine whether moderateintensity exercise is superior to vigorous-intensity exercise in the prevention of type 2 diabetes, and whether it is similar to diet/exercise in effectiveness.

In summary, a high amount of moderate-intensity exercise achieved $79 \%$ of the glucose-lowering effect that was experienced by an intensive combined lifestyle intervention consisting of diet and exercise designed to effect a 7\% weight loss over 6 months. In addition, moderate-intensity exercise was significantly superior to the energy-equivalent amount of vigorous-intensity exercise for improvements in glucose tolerance. Taken together with the numerous findings of strong independent relations between postprandial glucose levels and prediction of type 2 diabetes, these observations suggest that a high amount of moderate-intensity exercise may be a very effective intervention for preventing progression to diabetes in at-risk individuals. However, other than the significant difference in glucose tolerance, there were no other significant differences between the moderate- and vigorous-intensity exercise training groups for any other cardiometabolic healthrelated variable.

Acknowledgements We thank H. Cui, a Research Analyst II at Duke University, for her expert laboratory analyses in performing the immunoassays and clinical chemistry measurements for the blood analyses in the STRRIDE PD study. We thank L. Elliot Penry, our research nurse, for her hours of work in collecting, centrifuging and processing the numerous blood samples from the OGTT and screening bloods for STRRIDE-PD.

Funding The study was funded by National Institutes for Health National Institute of Diabetes and Digestive and Kidney Diseases (NIHNDDK) (R01DK081559).

Duality of interest The authors declare that there is no duality of interest associated with this manuscript.

Contribution statement CAS and WEK participated in conception and design, acquisition of data, data analysis, interpretation of data, and drafting and editing of the manuscript. LAB and LHW participated in conception and design, data collection, data interpretation and editing the manuscript. EOG, LWP and MJM participated in data collection, data interpretation and editing the manuscript. GPS, TLS, KMH and CWB participated in the conceptual design, data interpretation and editing the manuscript. $\mathrm{KMH}$ participated in data interpretation and editing the manuscript. All authors approved the final version. WEK is responsible for the integrity of the work as a whole.

\section{References}

1. Group DPPR (2002) Reduction in the incidence of type 2 diabetes with lifestyle intervention or metformin. New Engl J Med 346:393403

2. Bajpeyi S, Tanner C, Slentz C et al (2009) Effect of exercise intensity and volume on persistence of insulin sensitivity during training cessation. J Appl Physiol 106:1079-1085

3. Houmard J, Tanner C, Slentz C, Duscha B, McCartney J, Kraus W (2004) Effect of the volume and intensity of exercise training on insulin sensitivity. J Appl Physiol 96:101-106

4. Slentz C, Houmard J, Johnson J et al (2007) Inactivity, exercise training and detraining, and plasma lipoproteisn. STRRIDE: a randomized, controlled study of exercise intensity and amount. J Appl Physiol 103:432-442

5. Slentz C, Tanner C, Bateman L et al (2009) Effects of exercise training intensity on pancreatic beta cell function. Diabetes Care 32:1807-1811

6. Johnson J, Slentz C, Houmard J et al (2007) Exercise training amount and intensity effects on metabolic syndrome: STRRIDE. Am J Cardiol 100:1759-1767

7. Healy G, Dunstan D, Salmon J et al (2007) Objectively measured light-intensity physical activity is independently associated with 2-h plasma glucose. Diabetes Care 30:1384-1389

8. Jeon C, Lokken R, Hu F, van Dam R (2007) Physical activity of moderate intensity and risk of type 2 diabetes: a systematic review. Diabetes Care 30:744-752

9. Manders R, VanDijk J, VanLoon L (2010) Low-intensity exercise reduces the prevalence of hyperglycemia in type 2 diabetes. Med Sci Sports Exerc 42:219-225

10. Newsom S, Everett A, Hinko A, Horowitz J (2013) A single session of low-intensity exercise is sufficient to enhance insulin sensitivity into the next day in obese adults. Diabetes Care 36:2516-2522

11. Schenk S, Horowitz J (2007) Acute exercise increases triglyceride synthesis in skeletal muscle and prevents fatty acid-induced insulin resistance. J Clin Invest 117:1690-1698

12. Venables M, Jeukendrup A (2008) Endurance training and obesity: effect on substrate metaoblism and insulin sensitivity. Med Sci Sports Exerc 40:495-502

13. Kraus W, Houmard J, Duscha B et al (2002) Effects of the amount and intensity of exercise on plasma lipoproteins. New Engl J Med 347:1483-1492

14. Ross R, Hudson R, Stotz P, Lam M (2015) Effects of exercise amount and intensity on abdominal obesity and glucose tolerance in obese adults: a randomized trial. Ann Intern Med 162:325-334

15. Howley ET (2001) Type of activity: resistance, aerobic and leisure versus occupational phyiscal activity. Med Sci Sports Exerc 33: S364-S369

16. Matsuda M, Defronzo R (1999) Insulin sensitivity indices obtained from oral glucose tolerance testing: comparison with the euglycemic clamp. Diabetes Care 22:1462-1470

17. Kraus W, Torgan C, Duscha B et al (2001) Studies of a targeted risk reduction intervention through defined exercise (STRRIDE). Med Sci Sports Exerc 33:1774-1784

18. Duscha B, Slentz C, Johnson J et al (2005) Effects of exercise training amount and intensity on peak oxygen comsumption in middle-aged men and women at risk for cardiovascular disease. Chest 128:2788-2793

19. Brunner E, Shipley M, Witte D, Fuller J, Marmot M (2006) Relation Between Blood Glucose and Coronary Mortality Over 33 Years in the Whitehall Study. Diabetes Care 29:26-31

20. Rijkelijkhuizen J, Nijpels G, Heine R, Bouter L, Stehouwer C, Dekker J (2007) High risk of cardiovascular mortality in individuals with impaired fasting glucose is explained by conversion to diabetes: The Hoorn Study. Diabetes Care 30:332-336 
21. Marini M, Succurro E, Frontini S et al (2012) Insulin sensitivity, $\beta$-cell function, and incretin effect in individuals with elevated 1hour postload plasma glucose levels. Diabetes Care 35:868-872

22. de Vegt F, Dekker J, Ruhé H et al (1999) Hyperglycaemia is associated with all-cause and cardiovascular mortality in the Hoorn population: The Hoorn Study. Diabetologia 42:926-931

23. Smith N, Barzilay J, Shaffer D et al (2002) Fasting and 2-hour postchallenge serum glucose measures and risk of incident cardiovascular events in the elderly: The Cardiovascular Health Study. Arch Intern Med 162:209-216

24. Qiao Q, Pyörälä K, Pyörälä M et al (2002) Two-hour glucose is a better risk predictor for incident coronary heart disease and cardiovascular mortality than fasting glucose. Eur Heart J 23:1267-1275

25. Meigs J, Nathan D, D'Agostino RS, Wilson P, Framingham Offspring Study (2002) Fasting and Postchallenge Glycemia and Cardiovascular Disease Risk: the Framingham Offspring Study. Diabetes Care 25:1845-1850

26. Tominaga M, Eguchi H, Manaka H, Igarashi K, Kato T, Sekikawa A (1999) Impaired glucose tolerance is a risk factor for cardiovascular disease, but not impaired fasting glucose. The Funagata Diabetes Study. Diabetes Care 22:920-924

27. Group C (1999) Glucose tolerance and mortality: comparison of WHO and ADA diagnostic criteria. The DECODE study group.
European Diabetes Epidemiology Group. Diabetes Epidemiology. Collaborative analyses of diagnostic criteria in Europe. Lancet 354: 617-621

28. Abdul-Ghani M, DeFronzo R (2009) Plasma glucose concentration and prediction of future risk of type 2 diabetes. Diabetes Care 32: S194-198

29. Abdul-Ghani M, Lyssenko V, Tuomi T, DeFronzo R, Groop L (2009) Fasting versus postload plasma glucose concentration and the risk for future type 2 diabetes. Results from the Botnia study. Diabetes Care 32:281-286

30. Abdul-Ghani M, Williams K, DeFronzo R, Stern M (2007) What is the best predictor of future type 2 diabetes? Diabetes Care 30:1544 1548

31. Abdul-Ghani M, Stern M, Lyssenko V, Tuomi T, Groop L, DeFronzo R (2010) Minimal contribution of fasting hyperglycemia to the incidence of type 2 diabetes in subjects with normal 2-h plasma glucose. Diabetes Care 33:557-561

32. Abdul-Ghani M, Abdul-Ghani T, Stern M et al (2011) Two-step approach for the prediction of future type 2 diabetes risk. Diabetes Care 34:2108-2112

33. Nathan D, Davidson M, DeFronzo R et al (2007) Impaired fasting glucose and impaired glucose tolerance: implications for care. Diabetes Care 30:753-759 